\title{
Conceituando a Percepção em Sistemas Colaborativos: A Busca por Sistemas Sensíveis a Percepção
}

\author{
Max Felipe Silva Dos Santos Cravo ${ }^{1}$, Romeu Alves de Sousa ${ }^{1}$, Beatriz Saburido \\ Racca $^{1}$, Juliana Baptista dos Santos França ${ }^{1,2}$ \\ ${ }^{1}$ Departamento de Computação (DECOMP) - Universidade Federal Rural do Rio de \\ Janeiro (UFRRJ), Seropédica - RJ - Brazil \\ ${ }^{2}$ PPGMMC e PPGE - UFRRJ - Seropédica, RJ, Brasil \\ \{maxcravo25, romeualves1985, saburido57, julibsf\}@gmail.com
}

\begin{abstract}
Considering the human factor in collaborative systems field, this research seeks to understand the perception of collaborative interactions in computational processes. With the increase in the use of collaborative systems in personal, study and work relationships, perception has become the key point to be studied, so that through its understanding and conceptualization it is possible to design systems that are increasingly sensitive to perception. This article discusses the first steps of perception's conceptual model and defines the next steps of the research that seeks to consolidate quality requirements with a focus on perception.
\end{abstract}

Resumo. Considerando o fator humano na área de sistemas colaborativos, esta pesquisa busca a compreensão da percepção sobre as interações colaborativas em processos computacionais. Com o aumento da utilização de sistemas colaborativos nas relações pessoais, de estudo e trabalho, a percepção tem se tornado o ponto chave a ser estudado, de modo que através de sua compreensão e conceitualização seja possível projetar sistemas cada vez mais sensiveis à percepção. Este artigo discute os primeiros passos da construção de um modelo conceitual de percepção e define os próximos passos da pesquisa que busca a consolidação de requisitos de qualidade com o foco na percepção.

\section{Introdução}

Atualmente, temos observado a necessidade por sistemas cada vez mais inteligentes. Para isso, iniciativas que tornem os resultados computacionais e seu processamento mais próximos do raciocínio humano tem sido estimulada e realizada. A necessidade por estes sistemas tem sido mostrada haja visto o volume cada vez maior de dados que precisam ser processados, originado pelo conteúdo popular produzido na internet e pelo conhecimento de especialistas; sistemas especialistas que exigem cada vez mais refinamento em seus resultados; sistemas de suporte a decisão em domínios complexos que precisam cada vez mais de resultados sensíveis ao raciocínio humano e sua percepção, e transparência introduzida nos sistemas governamentais [Orasanu et al. 1993], [Qudrat-Ullah 2015], [França et al. 2017].

Esta necessidade trouxe à cena o emprego de sistemas colaborativos a fim de que o conhecimento explícito pudesse ser gerido e o conhecimento tácito pudesse ser transformado em explícito para que o valor pudesse ser incorporado ao objeto fim. No entanto, muito se tem discutido a partir dos três pilares da colaboração, sendo eles: 
comunicação, cooperação e coordenação [Fuks et al. 2008], mas pouco tem-se investido na compreensão e aplicabilidade da percepção no que tange as necessidades de negócio e sua tradução em resultados pelos sistemas computacionais. A aplicação dos sistemas colaborativos é defendida devido a captura de diferentes pontos de vista sobre uma questão, o que considerando domínios complexos, auxilia na gestão do conhecimento envolvido. Neste cenário, sistemas de suporte ao gerenciamento de questões complexas, como aqueles de suporte a decisão, tem seus resultados pautados em análises simplistas, que poderiam ser detalhadas em soluções mais robustas se os sistemas tivessem preparo para atuar sobre a percepção do usuário. Para isso, estratégias de suporte ao usuário para que ele possa melhor perceber as informações disponibilizadas são de fundamental importância.

A percepção que permeia as interações colaborativas, em sistemas ou processos computacionais, traz consigo novos parâmetros de cooperação [Collazos et al. 2019]. Vieira (2017) reforça a importância da percepção no modelo 3C [Fuks et al. 2003] e Fuks et al. (2011) coloca a percepção em foco no desenvolvimento de um sistema colaborativo. Para Fuks et al. (2011), a percepção tem como benefício à redução dos conflitos, duplicidade do trabalho, evita o isolamento em trabalhos individuais, amplia memória do grupo e permite medir a qualidade do próprio trabalho. Ela é um aspecto que está além de ser um coadjuvante no modelo $3 \mathrm{C}$, mas sim é responsável por exercer um papel complementar na interação colaborativa.

Segundo Collazos (2019), a percepção muitas das vezes atuou nas interações colaborativas de forma marginal. No entanto, com o advento do world wide web e o aumento no consumo de eletrônicos, observa-se uma ressurgência no interesse de sistemas perceptivos tanto em pesquisas quanto na área comercial. Considerando não só a crescente natural por sistemas colaborativos, em 2020 foi percebido o papel fundamental desses sistemas nas práticas sociais, bem como a necessidade por funcionalidades mais robustas no que se relaciona com a percepção do usuário. Neste ano de pandemia, profissionais e indivíduos distantes ou não das práticas tecnológicas se reinventaram introduzindo ferramental colaborativo em seu dia a dia [Vivacqua et al. 2020].

Dessa maneira, esse artigo tem por objetivo principal traçar o desenho de pesquisa quanto a conceitualização da percepção em sistemas colaborativos e promover ações que viabilizem a construção de sistemas sensíveis a percepção. Para isso, será apresentado neste artigo a primeira versão do modelo conceitual de percepção originado a partir de uma análise bibliográfica, detalhando seu processo de construção. Neste artigo também serão apresentadas as próximas fases da pesquisa e como ela será avaliada.

Este artigo está organizado em quatro seções. A primeira apresenta a introdução do artigo com os objetivos a serem alcançados e motivação para a pesquisa. A seção 2 apresenta a fundamentação da pesquisa e os trabalhos relacionados, enquanto a seção 3 apresenta o desenho de pesquisa, seu status e a primeira versão do modelo conceitual da percepção. Por fim, a seção 4 discute os próximos passos da pesquisa e as conclusões desse artigo.

\section{Fundamentação e Trabalhos Relacionados}

"Percepção é uma ação ou efeito de compreender o sentido de alguma coisa por meio das sensações ou da inteligência [Percepção 2020]". Trata-se de uma intuição geralmente 
de teor moral e avaliação sobre coisas ou seres a partir de um julgamento ou opinião. Relacionar a inteligência do indivíduo e suas sensações aos sistemas de computacionais de informação não é uma tarefa fácil. Com essa união busca-se por interações colaborativas com base computacional que estejam coerentes com a sensibilidade, inteligência e experiência do indivíduo ou do grupo de usuários.

Esta investigação está apoiada em quatro estudos principais. Na pesquisa de Nicolaci e Pimentel (2011), são caracterizadas as transformações decorrentes da Revolução da Internet e são realizadas análises sobre os sistemas colaborativos considerando as características da nova sociedade em rede e do novo ser humano digital que apresenta necessidade de suporte a sua percepção. Já nos estudos de [Fuks et al. 2011] são discutidas as teorias e modelos sobre a colaboração, fundamentando $\mathrm{o}$ desenvolvimento de sistemas colaborativos e permitindo selecionar e projetar esses sistemas em função de teorias e modelos.

Para Fuks et al. (2011) as teorias e modelos de colaboração, apresentam uma visão de como e porque as pessoas trabalham em grupo. Nesta pesquisa os autores discutem o modelo 3C de colaboração e explora este conceito em três dimensões: comunicação, coordenação e cooperação. Segundo eles "a comunicação é caracterizada pela troca de mensagens, pela argumentação e pela negociação entre pessoas; a coordenação é caracterizada pelo gerenciamento de pessoas, atividades e recursos; e a cooperação é caracterizada pela atuação conjunta no espaço compartilhado para a produção de objetos ou informações". Do ponto de vista de Fuks os elementos que compõem o modelo 3C precisam se inter-relacionar para que haja colaboração efetiva. É por meio das informações de percepções que o indivíduo obtém um feedback de suas próprias ações e feedthrough das ações de seus colegas.

$\mathrm{Na}$ investigação de Vivacqua e Garcia (2012) é apresentada uma ontologia da colaboração onde, os 3C's (comunicação, cooperação e coordenação) são detalhados em uma representação gráfica, mapeando os conceitos envolvidos e suas relações. As autoras apresentam os principais conceitos inter-relacionados, subdividindo a ontologia em quatro partes: (i) formação de grupos, (ii) comunicação, (iii) coordenação e (iv) cooperação. No entanto, embora haja uma conceitualização sobre cada elemento do modelo 3C, não há uma representação clara e formalizada da conceitualização da Percepção nesta discussão. Já em [Vieira et al. 2011] observa-se uma discussão sobre o aspecto percepção frente às suas implicações no desenvolvimento sistemas colaborativos. Apesar disso, existe uma carência de uma representação dos conceitos envolvidos com a percepção e suas relações.

Diante aos trabalhos relacionados apresentados, fica clara a necessidade de uma representação conceitual e formalizada da percepção, a fim de compreender este aspecto envolvido na fundamentação dos sistemas colaborativos. Dessa maneira, espera-se que a compreensão da percepção favorecerá a construção de sistemas sensíveis a percepção do usuário. Na próxima seção, discutiremos o desenho dessa pesquisa, pontuando as fases aguardando início, em andamento e finalizadas até o momento.

\section{Desenho da Pesquisa}

Como apresentado até aqui, o principal objetivo desta investigação é estabelecer ações que favoreçam a compreensão da percepção frente aos sistemas colaborativos e propor ações que viabilizem a construção de sistemas colaborativos mais sensíveis a percepção. 
Sendo assim, esta pesquisa está organizada em três fases (Figura 1), detalhadas a seguir:

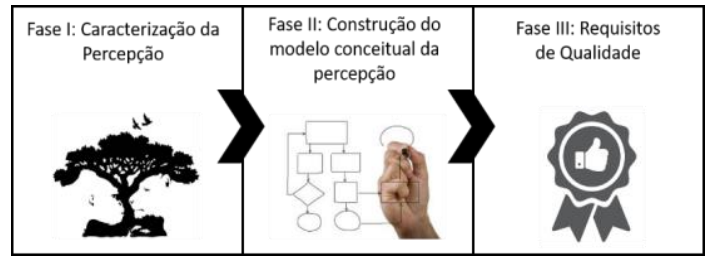

Figura 1: Desenho da pesquisa em macro visão.

Fase I. Definir um conjunto de conceitos caracterizadores da percepção: (a) Levantamento de conceitos através de referencial bibliográfico; (b) Levantamento de conceitos através de um survey com usuários de sistemas colaborativos;

Fase II. Desenvolver de forma incremental o modelo conceitual da percepção: (a) Construção de uma representação gráfica e formalizada do modelo conceitual, bem como o dicionário com a definição dos conceitos considerando os resultados alcançados na Fase I; (b) Atualização do modelo conceitual considerando os levantamentos bibliográficos e o survey com potenciais usuários de sistemas colaborativos; (c) Realizar investigações exploratórias com usuários de sistemas colaborativos a fim de confirmar ou refutar o modelo conceitual da percepção proposto;

Fase III. Propor requisitos de qualidade voltados à atuação da percepção em sistemas colaborativos: (a) Propor um método para levantamento e definição de requisitos não funcionais sensíveis à percepção; (b) Especificar ferramenta computacional colaborativa que permita interações colaborativas sensíveis a percepção; (c) Desenvolver ferramental tecnológico especificado; (d) Avaliar o método proposto e a ferramenta colaborativa sensíveis à percepção.

Sobre a metodologia de pesquisa aplicada, esta investigação se enquadra quanto a sua natureza como uma pesquisa qualitativa e de cunho exploratório. Qualitativa porque faz uso da abordagem para aprofundamento da investigação das questões que compõem o objeto de estudo e suas relações, valorizando o contato direto com a situação buscando elementos comum e permanecendo aberto para novas percepções. Exploratório porque busca-se ideias e intuições na tentativa de obter maior familiaridade com o objeto de estudo, permitindo aumentar o conhecimento dos pesquisadores e formular com maior precisão os problemas instanciados.

Até o momento as fases I e II estão em andamento, enquanto a fase III encontrase aguardando início. Para a fase i, o levantamento conduzido foi referente ao material bibliográfico estudado e, portanto, a primeira versão do modelo conceitual (Figura 2) ainda não considera o levantamento com os usuários de sistemas colaborativos. Quanto à evolução da fase II, será apresentada na subseção a seguir o processo de construção e a versão atual do modelo conceitual da percepção.

\subsection{Modelo Conceitual da Percepção}

Com a finalidade de construir um modelo conceitual da percepção, se faz necessário conceitualizar os aspectos e relações que define este termo. Nesta fase da pesquisa foi realizada uma revisão da literatura sobre o tema Percepção no contexto dos Sistemas 
Colaborativos no Google Scholar ${ }^{1}$. Os artigos resultantes desta busca, bem como o Livro de Sistemas Colaborativos (Fuks and Pimentel 2011) foram analisados pelos autores deste artigo e os conceitos que definem o termo percepção foram listados e definidos. Com base nessas definições, foram estabelecidas as relações entre os conceitos, construindo assim a primeira versão do modelo conceitual da percepção (Figura 2).

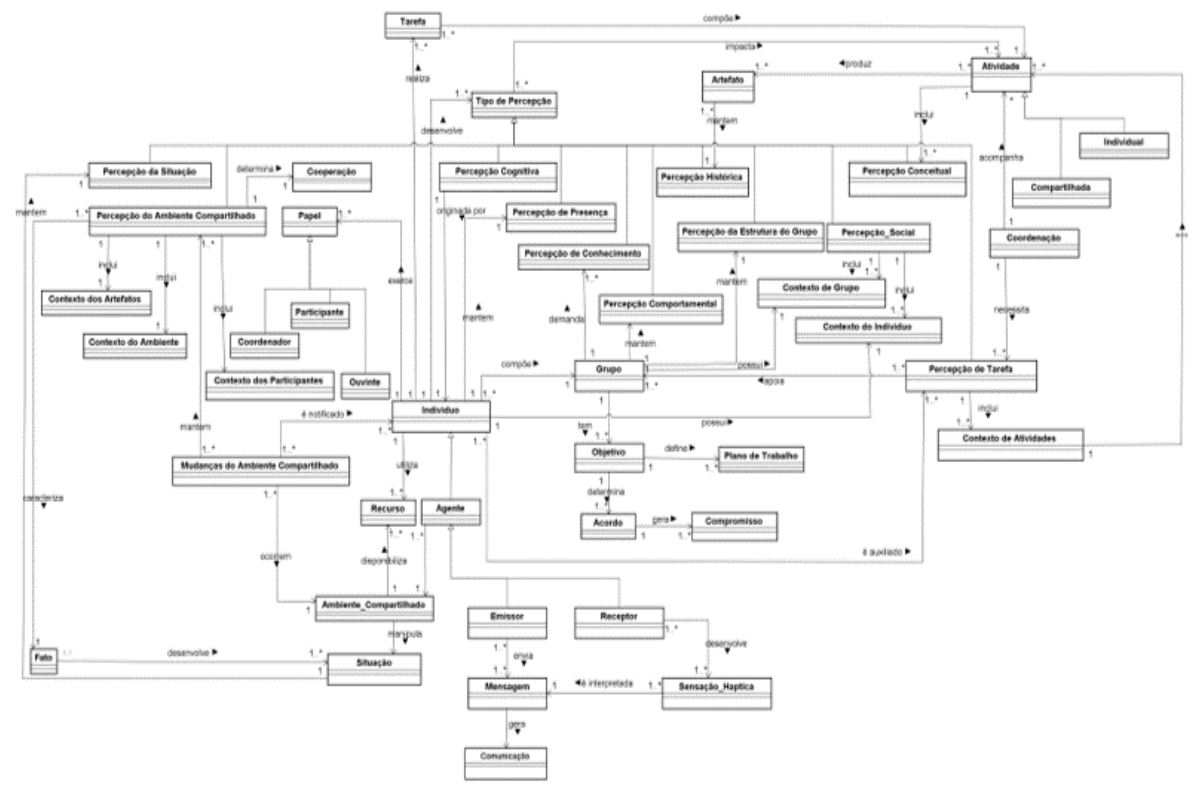

Figura 2: Modelo conceitual da percepção em sistemas colaborativos.

Conforme observado na Figura 2, o modelo apresentado foi construído em UML ${ }^{2}$. Nele são apresentados os principais conceitos que definem a Percepção, destacando e não se limitando a: (i) os tipos de percepção - cognitiva, comportamental, conceitual, do contexto, da estrutura do grupo, de presença, da situação, de tarefa, do ambiente compartilhado, do conhecimento, histórica e social; (ii) conceitos associados ao contexto - contexto de atividades, contexto de grupo, contexto de participante, contexto de artefatos; e (iii) conceitos associados à colaboração: grupo, participante, agente, papel, mensagem, informação, objetivo, plano de trabalho, coordenador, atividade, recursos, regras, tarefas entre outros conceitos.

\section{Conclusões e Próximos Passos da Pesquisa}

A percepção no contexto de sistemas colaborativos está associada à capacidade mental de um indivíduo compreender as ações executadas por outros membros de seu grupo de trabalho. Tal percepção, provê suporte ao participante para suas próprias atividades, minimizando sensações de solidão e potencializando as práticas do grupo.

A conceitualização da percepção em sistemas colaborativos foi iniciada com base no levantamento dos aspectos que definem a percepção em referencial bibliográfico e na proposição da primeira versão do modelo conceitual da percepção (Figura 2). O próximo passo dessa investigação é avaliar a versão atual do modelo conceitual da percepção junto aos pesquisadores da área de sistemas colaborativos e potenciais usuários desses sistemas.

\footnotetext{
${ }^{1}$ https://scholar.google.com.br/

${ }^{2}$ https://www.uml.org/what-is-uml.htm
} 
Espera-se que nesta avaliação ocorra a instanciação do modelo conceitual da percepção pelos participantes do estudo, considerando um cenário exemplo formulado pelos autores da pesquisa.

Para as próximas fases dessa investigação é esperada desde a atualização do modelo conceitual proposto, a construção do método para levantamento e definição de requisitos não funcionais de percepção, até a avaliação dos artefatos produzidos seguindo o desenho de pesquisa apresentado na seção 3 .

\section{Agradecimentos}

Esta pesquisa recebeu apoio da FAPERJ - E-26/211.367/2019 (248406).

\section{Referências}

Collazos, C. A., Gutiérrez, F. L., Gallardo, J., Ortega, M., Fardoun, H. M., \& Molina, A. I. (2019). Descriptive theory of awareness for groupware development. Journal of Ambient Intelligence and Humanized Computing, 10(12), 4789-4818.

França, J. B. S., Neiva, F. W., Dias, A. F. S., \& Borges, M. R. S. (2017). Toward impact projection characterization of complex decisions. In IEEE International Conference on Systems, Man, and Cybernetics, 2017, Banff. IEEE International Conference on Systems, Man, and Cybernetics (Vol. 1, pp. 1-6).

Fuks, H., Raposo, A. B., Gerosa, M. A., \& Lucena, C. J. P. (2003). Do modelo de colaboração 3c à engenharia de groupware. Simpósio Brasileiro de Sistemas Multimídia e Web-Webmidia, 0-8.

Fuks, H., Raposo, A., \& Gerosa, M. A. (2008). The 3c collaboration model. In Encyclopedia of Ecollaboration (pp. 637-644). IGI Global.

Fuks, H.; Raposo, A.; Gerosa, M. A.; Pimentel, M.; Filippo, D. and Lucena, C. J. P. (2011) “Teorias e Modelos de Colaboração" In Pimentel, M. and Fuks, H. (Org.). Sistemas Colaborativos. Rio de Janeiro: Elsevier. cap. 2, p. 16-33.

Nicolaci-da-Costa, A. M. and Pimentel, M. (2011) "Sistemas Colaborativos para uma nova sociedade e um novo ser humano" In: Pimentel, M. and Fuks, H. (Org.). Sistemas Colaborativos. Rio de Janeiro: Elsevier. cap. 1, p. 3-15.

Orasanu, J., \& Connolly, T. (1993). The reinvention of decision making. Decision making in action: Models and methods, 1, 3-20.

Percepção. In: Dicio, Dicionário Online de Português. 2020. Disponível em: $<$ https://www.dicio.com.br/percepcao/>. Acesso em: 23/12/2020.

Pimentel, M., \& Fuks, H. (Eds.). (2012). Sistemas colaborativos. Elsevier.

Qudrat-Ullah, H. (2015). Better Decision Making in Complex, Dynamic Tasks. Training with HumanFacilitated. Interactive Learning Environments. School of Administrative Studies. York University, Toronto, 159-177.

Vieira, V.; Tedesco, P.; Salgado, A.C. (2012). Percepção e Contexto. In: Pimentel, M.; Fuks, H. Sistemas colaborativos. Rio de Janeiro: Editor Elsevier

Vivacqua, A.; Garcia, A.C. (2012) Ontologia de Colaboração. In: Pimentel, M.; Fuks, H. Sistemas colaborativos. Rio de Janeiro: Editor Elsevier

Vivacqua, A.S.; Machado, L.; Vieira, V. (2020) Colaboração em Tempos de Pandemia: I Workshop de Colaboração Online. SBC Horizontes. ISSN: 2175-9235. Disponível em: $<$ http://horizontes.sbc.org.br/?p=4294>. Acesso em: 15 out. 2020 